Published in "World Neurosurgery 137(): 84-88, 2020"

which should be cited to refer to this work.

\title{
Anatomic Analysis of the Internal and External Aspects of the Pterion
}

\author{
Aysun Uz ${ }^{1,3,4}$, Ali Can Korkmaz ${ }^{1}$, Luis Filgueira ${ }^{3}$, Mehmet Ali Guner ${ }^{1,5}$, Richard Shane Tubbs ${ }^{6,7}$, \\ Ayse Karatas Demirciler ${ }^{2,8}$
}

OBJECTIVE: The pterion is an H-shaped suture complex. This study's goal was to determine the location of its external and internal surfaces and extension and emphasize and discuss its surgical importance.

METHODS: Fifty dried adult human skulls were obtained from the Department of Anatomy. A 2-mm drill bit was placed externally over the pterion, and the pterion was drilled through the bone perpendicular to the skull's surface.

RESULTS: The midpoint of the $H$ shape in the pterion area was not at the same level on the skull's external and internal pterion surfaces. According to these measurements, the external pterion lay above the internal pterion when the skull was viewed externally. Furthermore, the internal pterion was on average longer than the external pterion. The internal and external pterions were schematized such that the skull was viewed from the outside. These areas were divided into 4 quadrants (anterior-superior, anterior-inferior, posterior-superior, and posteriorinferior) by a vertical and horizontal line. In 30 cases $(60 \%)$, sulci of the middle meningeal artery's parietal branches entered the posterior-superior quadrant on the bone, whereas the artery's frontal branches were located in the anterior-superior and anterior-inferior quadrants, and the Sylvian fissure's origin was in the posterior-inferior quadrant.
CONCLUSIONS: By using a subdivision into 4 quadrants, and considering our anatomic findings, we determined the way surgical procedures can be performed more easily and reliably. Even with modern localization technologies, anatomic landmarks can be useful to the neurosurgeon.

\section{INTRODUCTION}

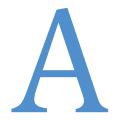
ccurate location of burr holes is important in neurosurgery. A safe surgical approach begins with a good understanding of superficial anatomic landmarks, craniometric points that are used in the preoperative calculations. The surgeon relies on external landmarks, such as the pterion, which is one of the landmarks in the neurosurgeon's armamentarium used most commonly to perform an appropriate craniotomy. Pterional craniotomy (also referred to as a frontotemporal, or frontotemporosphenoidal craniotomy) is one used most widely in neurosurgical practice because it provides wide frontobasal exposure while minimizing brain retraction. The pterional approach allows the surgeon to access the middle cranial fossa, the anterior cranial fossa, the suprasellar and parasellar structures, and the circle of Willis. ${ }^{\mathrm{I}-9}$ The pterion is an H-shaped suture complex $^{\text {IO }}$ in which the parietal and sphenoid bones form the midpoint of the $\mathrm{H}$. Although the anterior limb of the $\mathrm{H}$ shape forms sutures between the frontal, parietal, and sphenoid bones, the posterior limb is composed of the sutures between

\footnotetext{
Key words

- Anatomy

- Middle meningeal artery

- Pterion

- Sylvian fissure
}

\section{Abbreviations and Acronyms}

Pe: External pterion

Pea: Anterior of external pterion

Pep: Posterior of external pterion

Pi: Internal pterion

Pia: Anterior of internal pterion

Pip: Posterior of internal pterion

From the Departments of ${ }^{\mathbf{1}}$ Anatomy and ${ }^{\mathbf{2}}$ Neurosurgery, Ankara University School of Medicine, Morfoloji, Sihhiye, Ankara, Turkey; ${ }^{3}$ Department of Anatomy, Faculty of Science and Medicine, University of Fribourg, Fribourg, Switzerland; ${ }^{4}$ Department of Neuroscience, Ankara University Graduate School of Health Science, Ankara, Turkey; ${ }^{\mathbf{5}}$ Department of Anatomy, University of Health Sciences Faculty of Medicine, Etlik, Ankara, Turkey;

${ }^{6}$ Department of Neurosurgery, Tulane University School of Medicine, New Orleans, Louisiana, USA; 'Department of Anatomical Sciences, St. George's University, Grenada, West Indies; and ${ }^{\boldsymbol{8}}$ Department of Neurosurgery, Izmir Katip Celebi University, Ataturk Education and Research Hospital, Izmir, Turkey

To whom correspondence should be addressed: Aysun Uz, M.D.

[E-mail: aysunuz99@yahoo.com] 
the parietal, temporal, and sphenoid bones. Therefore, it is more accurate to state that the pterion is an area between the frontal bone, the squamous part of the temporal bone, the sphenoidal bone's greater wing, and the parietal bone's sphenoidal angle. We examined the middle part of this suture complex, which consists of the parietal and sphenoid bones, to achieve this study's objective: to determine the location of this area outside and inside the skull and emphasize and discuss its surgical importance.

\section{MATERIALS AND METHODS}

Fifty dried adult human skulls were obtained from the Department of Anatomy. A 2-mm drill bit was placed externally over the pterion, and it was drilled through the bone perpendicular to the skull surface. The skulls were horizontally divided into 2 parts over the inferior temporal line as calvaria and cranial base. Then, the drill hole's position was determined on the inner surface, after which the midpoint of the $\mathrm{H}$-shaped suture was measured on the skull's external and internal surfaces. The front and back points of this area also were determined internally and externally.

The midpoint of this $\mathrm{H}$-shaped region is referred to as the internal pterion (Pi) and the anterior of internal pterion (Pia) and posterior of internal pterion (Pip) points of the Pi were determined. The anterior of external pterion (Pea) and posterior of external pterion (Pep) points were determined on the external skull surface. The external pterion's (Pe) anterior and posterior points were drilled, their location on the skull's internal surface was examined, and the distances between Pi and Pe were measured.

\section{RESULTS}

The H-shape's midpoint was not at the same level on the skull's external and internal surfaces (Figure 1). Therefore, these areas' distances from the inferior temporal line were measured on both the skull's external and internal surfaces based on both pterion midpoints (Figure 2). The distance between the Pe and

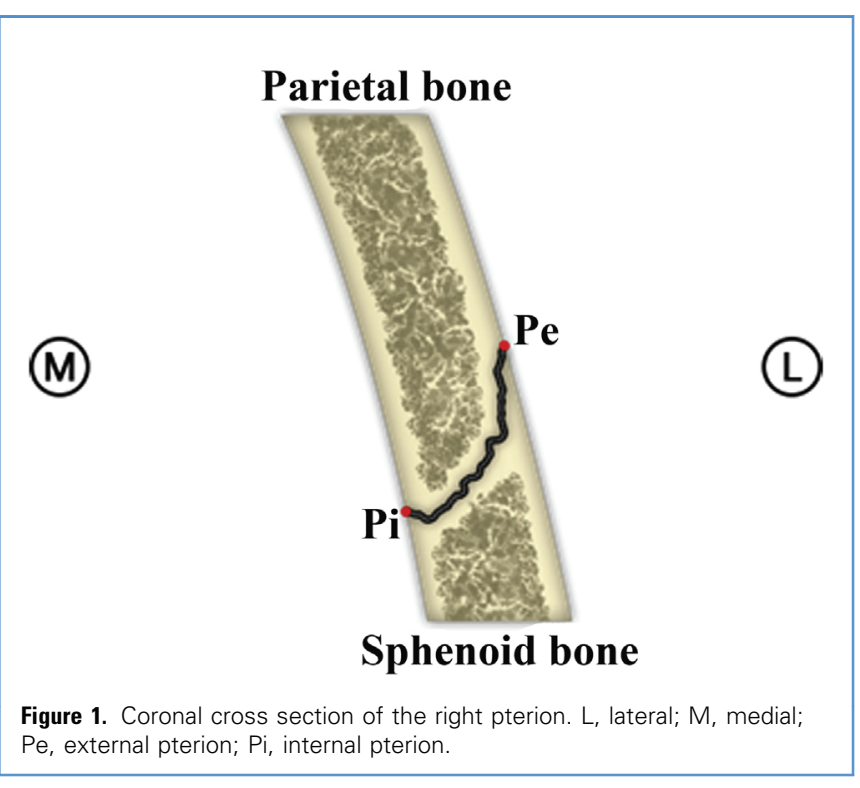

\section{Inferior Temporal Line

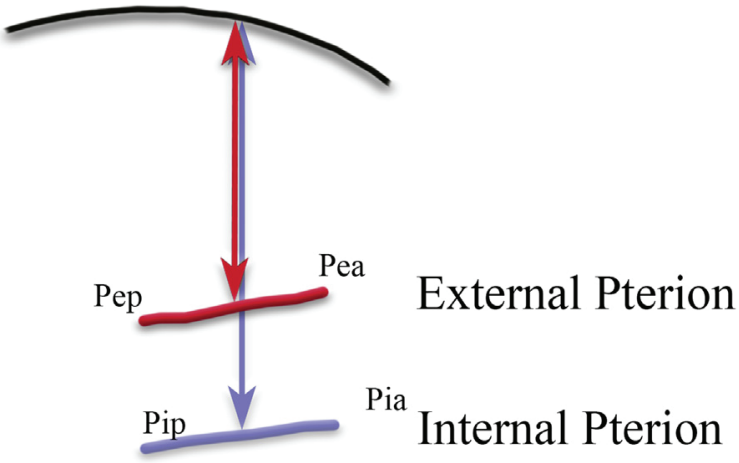

Figure 2. Right external and internal pterion on the skull as an illustration. The distance between the midpoint of the external pterion and the inferior temporal line is indicated as the red vertical line. The distance between the midpoint of the internal pterion and the inferior temporal line is indicated as the blue vertical line. Pea, anterior of the external pterion; Pep, posterior of the external pterion; Pia, anterior of the internal pterion; Pip, posterior of the internal pterion.

the inferior temporal line was $14.50 \pm 2.04 \mathrm{~mm}$ on average, whereas the distance between the Pi and the inferior temporal line was $2 \mathrm{I} .8 \mathrm{o} \pm 2.64 \mathrm{~mm}$ on average (Table 1). According to these measurements, the Pe was approximately $6.3 \mathrm{~mm}$ superior to the Pi when the skull was viewed externally.

The drill-placed Pep penetrated the middle meningeal artery's parietal branches in 30 of 50 cases $(60 \%)$. In 12 cases $(24 \%)$, the grooves of these branches passed superior to the Pep, whereas in 8 cases ( $16 \%)$, they passed inferior to the Pep.

Comparing the distance between the anterior and posterior of the midpoint of the $\mathrm{H}$ shape on the Pi and Pe, the distance between the Pia and Pip was ro.84 $\pm 3.30 \mathrm{~mm}$, whereas that between the Pea and Pep was $8.88 \pm 3.05 \mathrm{~mm}$. Therefore, Pi was approximately I.96 $\mathrm{mm}$ longer on average than Pe (Table 1, and Figures 3 and 4).

The posterior part of the Pi was located at the Sylvian fissure's origin between the frontal and temporal lobes. The Sylvian fissure

Table 1. Measurements of the Internal Pterion and External Pterion

\begin{tabular}{|l|r|r|}
\hline Measurement & Mean \pm SD (mm) & Range (mm) \\
\hline $\begin{array}{l}\text { Vertical distance between the } \\
\text { midpoint of the internal petrion } \\
\text { area and inferior temporal line }\end{array}$ & $21.80 \pm 2.64$ & $15.72-27.40$ \\
\hline $\begin{array}{l}\text { Vertical distance between the } \\
\text { midpoint of the external petrion } \\
\text { area and inferior temporal line }\end{array}$ & $14.50 \pm 2.04$ & $10.4-17.52$ \\
\hline Distance between Pia and Pip & $10.84 \pm 3.30$ & $5.58-18.20$ \\
\hline Distance between Pea and Pep & $8.88 \pm 3.05$ & $3.47-17.49$ \\
\hline
\end{tabular}

Pia, anterior of the internal pterion; Pip, posterior of the internal pterion; Pea, anterior of the external pterion; Pep, posterior of the external pterion. 


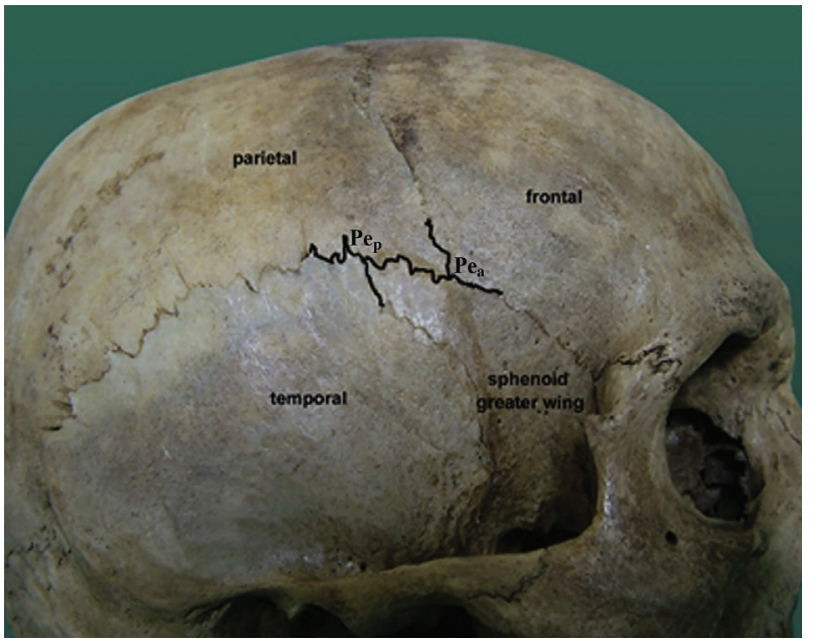

Figure 3. Anatomic position of the right external pterion. $\mathrm{Pe}_{\mathrm{a}}$, anterior of the external pterion, $\mathrm{Pe}_{\mathrm{p}}$, posterior of the external pterion.

separates the frontal and parietal lobes superiorly from the temporal lobe inferiorly. Intracranially, the pterion is an important landmark overlying both the middle meningeal artery's frontal branch and the Sylvian fissure. The middle meningeal artery's frontal branch was located in the anterior part of the Pi in $50 \%$ of cases and in the superior part in 50\% of cases.

The Pe and Pi areas are shown in Figures 5 and 6. These areas are divided into 4 quadrants (anterior-superior, anterior-inferior, posterior-superior, and posterior-inferior) by the vertical and horizontal lines. In 30 cases $(60 \%)$, the arterial sulci of the middle meningeal artery's parietal branches crossed the posteriorsuperior quadrant on the bone, whereas the frontal branches were located in the anterior-superior and anterior-inferior

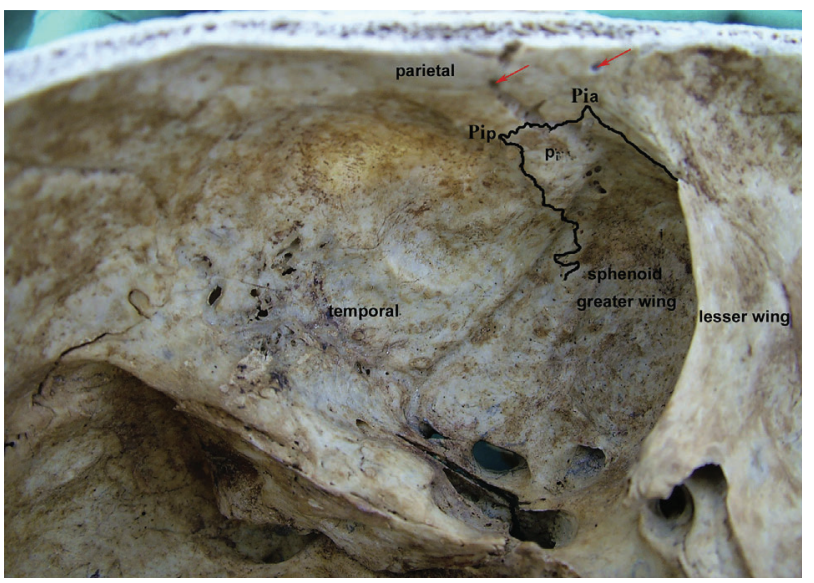

Figure 4. Anatomic position of the left internal pterion placed. The red arrows indicate the drill holes. Pia, anterior of the internal pterion; Pip, posterior of the internal pterion; $\mathrm{Pi}$, internal pterion. quadrants, and the Sylvian fissure's origin was in the posteriorinferior quadrant.

\section{DISCUSSION}

The pterional approach has been the transcranial approach used most commonly in neurosurgery because of its efficiency and familiarity to neurosurgeons. Frontotemporal approaches were developed and used for most of the 2oth century; however, Yasargil et al. ${ }^{\mathrm{II}}$ described and popularized the first modern pterional approach in 1976 . This technique involves approaching the brain and the anterior skull base through the lateral aspect by removing the frontal and temporal bones and greater sphenoid wing. ${ }^{\text {II }}$ The key steps in pterional craniotomy are drilling the sphenoid wing as far down as the superior orbital fissure, with or without removing the anterior clinoid, and opening the Sylvian fissure and basal cisterns. Tumoral and vascular lesions that involve the sellar/parasellar area, anterior and anterolateral circle of Willis, middle cerebral artery, anterior brainstem, upper basilar artery, insula, basal ganglia, mesial temporal region, anterior cranial fossa, orbit, and optic nerve are within reaching through the pterional approach. ${ }^{5, \text { I2 }}$

In pterional craniotomies, bleeding usually results from the sphenoid wing and middle meningeal artery when the bone flap is lifted. The sphenoid bone's lesser wing is drilled at the apex of the superior orbital fissure up to the level of the meningo-orbital (recurrent meningeal) artery, which provides wide exposure. Over past decades, the procedure has undergone certain modifications, including variable removal of the zygoma, sphenoid wing, and orbital roof, or performing clinoidectomy. In I996, Rhoton made a comment to the Day et al. study ${ }^{5}$ and emphasized that in frontotemporal craniotomies, in addition to removing the sphenoid ridge, the roof of the optic canal, and the lesser wing close to the orbital roof, proximal control is maintained over a large area allowing access to basilar apex aneurysms and not simply the anterior circulation. In addition, he stated that the anterior temporal lobe's mobility could be increased when necessary by removing the sphenoid wing's greater wing and the squamous part of the temporal bone toward the middle cranial fossa's base. ${ }^{3,5}$

Combined approaches and small craniotomies have been attempted to reduce complications during pterional and subtemporal procedures. In 1993, Heros and Lee ${ }^{\mathrm{I} 3}$ reported that the combined approach reduces the risk of surgical complications. The orbitozygomatic craniotomy is derived from the classic pterional approach. Sakovich et al. ${ }^{\mathrm{I}}$ reported the advantages of smaller craniotomies used in pterional approaches to intracranial aneurysms.

Knowledge of superficial anatomy during surgical approaches is important to surgeons' concentration and determining and planning the surgical strategy. ${ }^{5,7,15,16}$ The pterion is among the most important landmark in neurosurgery, and there are 2 clinically significant reasons for its popularity. First, extradural hematomas usually are caused by fractures of the bones that form the pterion. Second, this region is a common keyhole for anterior and middle cranial fossa surgeries. Both situations entail risks because they are in the artery's groove in the internal aspect of the skull in close proximity to the middle meningeal artery. The groove for the middle meningeal artery is present on the frontal and parietal bones. Therefore, many papers have discussed the relation 


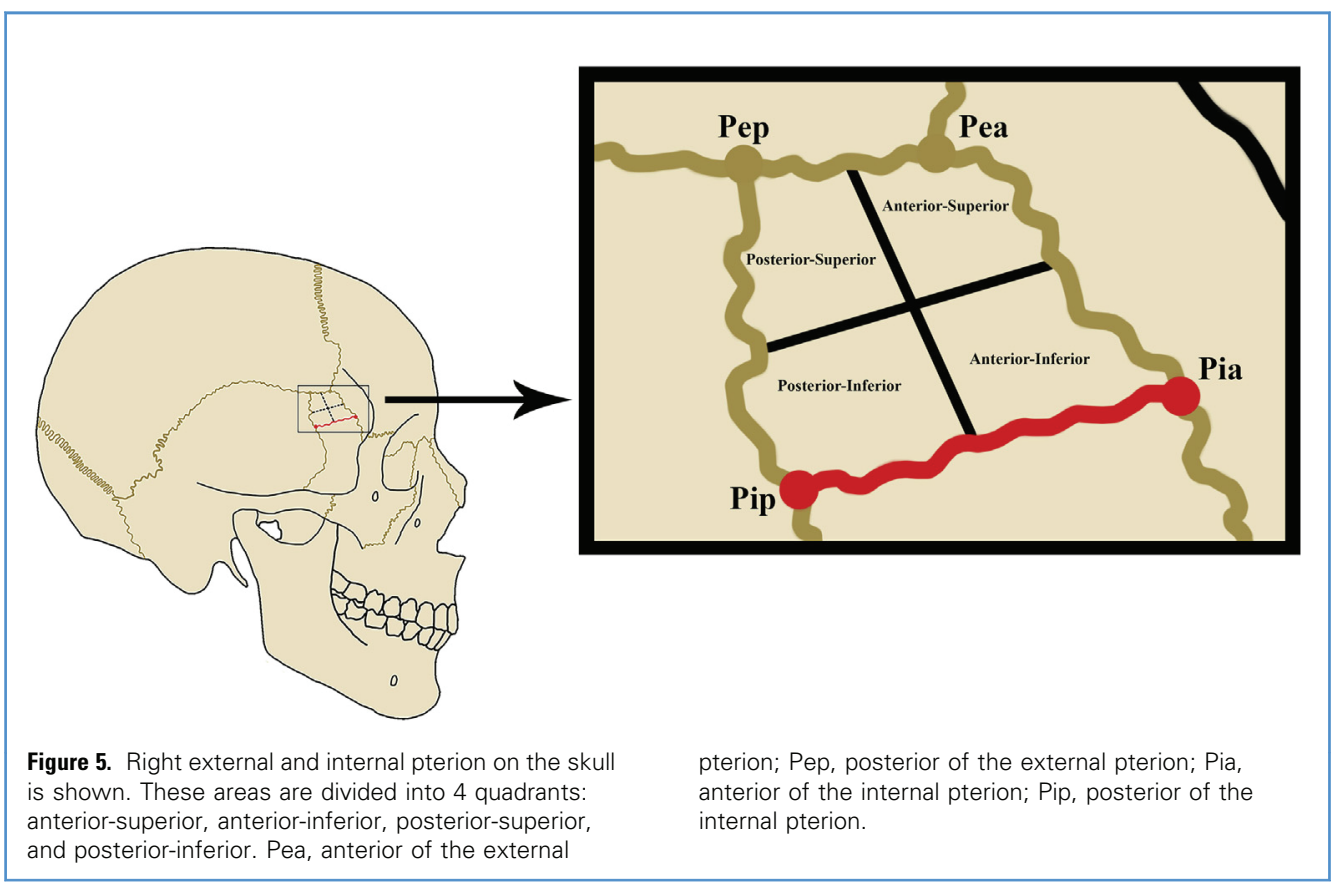

between the pterion and middle meningeal artery. ${ }^{17,18}$ In the Fujimoto et al. study, ${ }^{18}$ a bony canal for the middle meningeal artery was observed in the parietal bone $(34.9 \%)$ and sphenoid bone $(4.7 \%)$, at the intersection of the sphenoparietal suture and

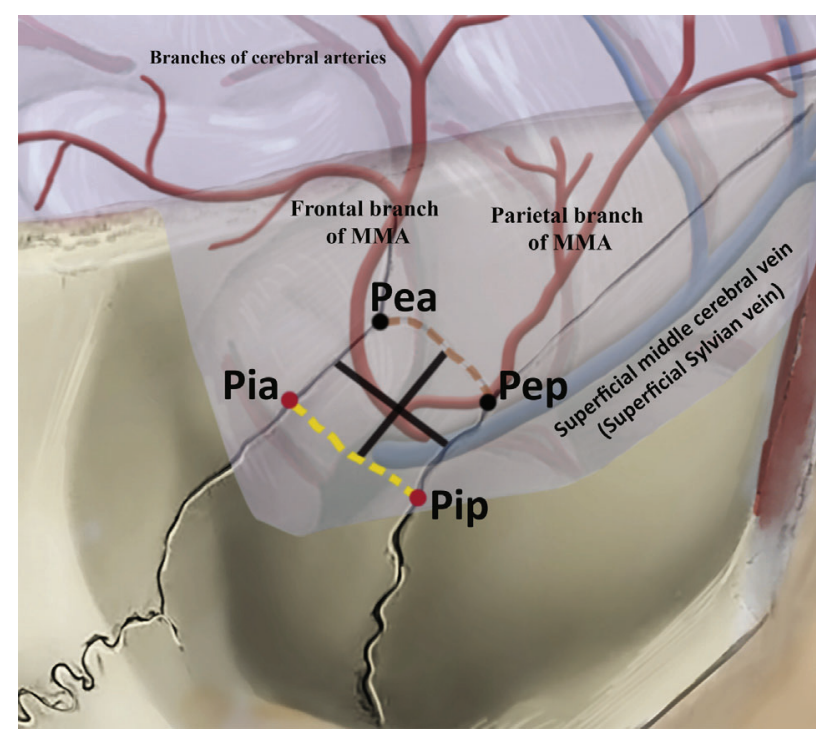

Figure 6. Four quadrants in between the external and internal pterions. The frontal and parietal branches of the middle meningeal artery and the superficial middle cerebral vein are demonstrated in these quadrants. The superficial middle cerebral vein is located on the Sylvian fissure. MMA, middle meningeal artery; Pea, anterior of the external pterion; Pep, posterior of the external pterion; Pia, anterior of the internal pterion; Pip, posterior of the internal pterion. coronal suture $(38 \%)$, and in the sphenofrontal suture (14.2\%), coronal suture $(6.3 \%)$, and sphenoparietal suture (I. $5 \%$ ). The authors concluded that the bony canal was located around the pterion. The Ma et al. study ${ }^{17}$ showed that the middle meningeal artery's frontal branch overlapped the pterion in $68 \%$ of 152 skulls ( 76 dried skulls). In our study, when applied to the Pe's posterior aspect, the drill penetrated the middle meningeal artery's parietal branches in $60 \%$ of the cases. In 12 cases $(24 \%)$, these branches' grooves were over the Pe's posterior point, whereas in 8 cases $(\mathrm{r} 6 \%)$ they passed under its posterior point. The anterior part of the $\mathrm{Pi}$ is the origin of the middle meningeal artery's frontal branch.

In our study, the Pe was $6.3 \mathrm{~mm}$ above the Pi areas when the skull was viewed externally (Figure 5). The Sylvian fissure, which begins at the Pi area's posterior point, often is used in surgical operations because by entering here, the lesser wing of the sphenoid and anterior clinoid process can be reached. Therefore, it provides a wide exposure for anterior circulation and basilar apex aneurysms (Figure 5).

As a result, using all the data obtained in this study allowed us to determine a way the surgical procedure can be performed more easily and reliably. When the skull is viewed from the outside, the $\mathrm{Pi}$ and Pe areas are divided into 4 quadrants, as shown in Figures 5 and 6 . The posterior-inferior part is the Sylvian fissure's origin and the superficial middle cerebral vein is located here. Therefore, the risks that can be associated with the superficial middle cerebral vein's bleeding should be considered. When the burr hole is opened, the riskiest areas are the anterior-inferior, anterior-superior, and posterior-superior parts. However, because of our findings, the middle meningeal artery's anterior and posterior branches can be found in these areas, and pterional craniotomies performed by considering these areas may reduce the risk of further subdural hematomas. Finally, even with modern 
localization technologies, anatomic landmarks can be useful to the neurosurgeon.

\section{CRedIT AUTHORSHIP CONTRIBUTION STATEMENT}

Aysun Uz: Conceptualization, Methodology, Formal analysis, Data curation, Writing - review \& editing. Ali Can Korkmaz: Formal analysis, Data curation, Writing - original draft. Luis Filgueira: Formal analysis, Data curation, Methodology, Writing - original draft. Mehmet Ali Guner: Writing - original draft. Richard Shane Tubbs: Writing - review \& editing, Methodology. Ayse Karatas Demirciler: Formal analysis, Data curation, Methodology, Writing - original draft, Writing - review \& editing.

\section{REFERENCES}

I. Tulleken CAF, Luiten MLFB. The basilar artery bifurcation in situ approached via the Sylvian route (50X): an anatomic study in human cadavers. Acta Neurochir (Wien). 1986;80:109-II5.

2. Solomon RA, Stein BM. Surgical approaches to aneurysms of the vertebral and basilar arteries. Neurosurgery. I988;23:203-208.

3. Tedeschi H, Rhoton AL. Lateral approaches to the petroclival region. Surg Neurol. I994;4I:I80-2I6.

4. Tanaka Y, Kobayashi S, Sugita K, Gibo H, Kyoshima K, Nagasaki T. Characteristics of pterional routes to basilar bifurcation aneurysm. Neurosurgery. I995;36:533-540.

5. Day JD, Kellogg JX, Tschabitscher M, Fukushima T. Surface and superficial surgical anatomy of the posterolateral cranial base: significance for surgical planning and approach. Neurosurgery. 1996;38:1079-1084.

6. Nutik SL. Pterional craniotomy via a transcavernous approach for the treatment of low-lying distal basilar artery aneurysms. J Neurosurg. I998; 89:92I-926.

7. Uz A, Ugur HC, Tekdemir I. Is the asterion a reliable landmark for the lateral approach to posterior fossa? J Clin Neurosci. 200I;8:I46-I47.

8. Zalawadia A, Vadgama J, Ruparelia S, Patel S, Rathod SP, Patel SV. Morphometric study of pterion in dry skull of Gujarat region. NJIRM. 20I0; I: $25-29$

9. Reis BL, Silveira RLD, Gusmao SNS. Sphenopterional point: strategic point for burr hole placement in frontotemporal craniotomies. World Neurosurg. 2017;105:399-405.

Io. Fitzgerald MJT, Gruner G, Mtui E. Clinical Neuroanatomy and Neuroscience. 6th ed. London, UK: Elsevier Saunders; 2012.

II. Yasargil MG, Antic J, Laciga R, Jain KK, Hodosh RM, Smith RD. Microsurgical pterional approach to aneurysms of the basilar bifurcation. Surg Neurol. 1976;6:83-9I.

12. Altay T, Couldwell WT. The frontotemporal (pterional) approach: an historical perspective. Neurosurgery. 20I2;7I:48I-492.

I3. Heros RC, Lee SH. The combined pterional/ anterior temporal approach for aneurysms of the upper basilar complex: technical report. Neurosurgery. I993;33:244-25I.

I4. Sakovich VP, Kolotvinov VS, Shamov A. The surgical treatment of intracranial aneurysms from the pterion approach using small trephining openings. Zh Vopr Neirokhir Im N N Burdenko. 2000;1:3-6 [discussion: 7].

I5. Yasargil MG. Microneurosurgery: Microsurgery of CNS Tumors. Vol. 4B. New York, NY: Thieme Medical Publishers; I995.
I6. Oguz O, Sanli SG, Bozkir MG, Soames RW. The pterion in Turkish male skulls. Surg Radiol Anat. 2004;26:220-224.

17. Ma S, Baillie LJM, Stringer MD. Reappraising the surface anatomy of the pterion and its relationship to the middle meningeal artery. Clin Anat. 2012;25: 330-339.

18. Fujimoto M, Otsuka N, Ezure H, Moriyama $\mathrm{H}$, Inoue $\mathrm{Y}$, Mori $\mathrm{R}$. Intracranial bony canal of the middle meningeal artery: morphological and histological analysis. Okajimas Folia Anat Jpn. 2017;93: IIQ-I25.

Conflict of interest statement: The authors declare that the article content was composed in the absence of any commercial or financial relationships that could be construed as a potential conflict of interest.

Received 16 October 2019; accepted 25 January 2020

Citation: World Neurosurg. (2020) 137:84-88. https://doi.org/10.1016/j.wneu.2020.01.198

Journal homepage: www.journals.elsevier.com/worldneurosurgery

Available online: www.sciencedirect.com

1878-8750/\$ - see front matter (C) 2020 Elsevier Inc. All rights reserved. 